\title{
General framework for adsorption processes on dynamic interfaces
}

\author{
Markus Schmuck \\ Maxwell Institute for Mathematical Sciences and Department of Mathematics, \\ Heriot-Watt University, Edinburgh, EH14 4AS \\ E-mail: m.schmuck@hw.ac.uk
}

\section{Serafim Kalliadasis}

Department of Chemical Engineering, Imperial College London, London SW7 2AZ, United Kingdom

E-mail: s.kalliadasis@imperial.ac.uk

\begin{abstract}
We propose a novel and general variational framework modelling particle adsorption mechanisms on evolving immiscible fluid interfaces. A by-product of our thermodynamic approach is that we systematically obtain analytic adsorption isotherms for given equilibrium interfacial geometries. We validate computationally our mathematical methodology by demonstrating the fundamental properties of decreasing interfacial free energies by increasing interfacial particle densities and of decreasing surface pressure with increasing surface area.
\end{abstract}

PACS numbers: $00.00,20.00,42.10$

Keywords: dynamic surface tension, gradient flow, complex adsorption

\section{Introduction}

Adsorption processes on interfaces are of great importance in a wide spectrum of scientific and industrial applications such as the stabilization of foams [1], the formulation of nanoporous materials, functional membranes and capsules, drug delivery $[2,3]$ and oil recovery [4], to name but a few. Also, many aerated food products such as bread cake, meringue, ice-cream and mousse are based on stabilized emulsions. Not surprisingly, (particle) adsorption has been an active topic of both experimental and theoretical research for several decades. Equally important are mixtures of different types of particles which require a dynamic description of the interfacial tension due to interactions between the different species involved, especially if one considers surfactants. In general, the interface between any two bulk neighbouring phases characterizes and mediates any physical process occuring between these two phases. Additional 
complexities include adsorption phenomena observed for charged particles [5], capping ligands [6], or swelling latex particles [7] on fluid-fluid interfaces.

Here, we develop a new and generic free-energy formalism for dynamic but diffuse interfaces in arbitrary spatial dimensions. So far, a small number of previous modelling attempts was restricted to a specific one-dimensional setting with a fixed interface $[8,9]$ or neglected fluid flow in an extension of $[8,9]$ to a diffuse interface formulation [10]. We incorporate the experimentally important characterization of particles by the contact angle into our mathematical description. Our derivation is based on well-established and rigorous mathematical results which connect the diffuse interface approach, i.e., the van der Waals/Cahn-Hilliard free-energy formalism, to the interfacial tension. This result is known as the Modica-Mortola theorem [11] and relates the interfacial tension to the homogeneous free energy, e.g. the classical and phenomenological double-well potential $W(s):=\left(1-s^{2}\right)^{2} / 4$.

Our formalism further generalizes the notions (e.g. employed in [12]) of microscopic surface tension $\sigma$, which describes a particle-free interface, as well as the macroscopic surface tension $\sigma_{A}$, which depends on the density $c$ of adsorbed particles, i.e., we can write the surface pressure $\Pi_{s}(c)$ from [12, equation (33)] in the more general form

$$
\Pi_{s}(\phi, c)=\sigma-\sigma_{A}=-\int_{\Omega} \mathrm{s}(\lambda) W(\phi) f_{I}(\phi, c) d \mathbf{x},
$$

since the particle-free interface is related to the perimeter functional $\sigma=\int_{\Omega} e(\phi) d \mathbf{x}$ and hence the macroscopic surface tension reads $\sigma_{A}=\int_{\Omega} e(\phi)+\mathrm{s}(\lambda) W(\phi) f_{I}(\phi, c) d \mathbf{x}$, see also (5), (6), and Figure 5 below. The variable $f_{I}(\phi, c)$ defines a specific interfacial free energy and $s(\lambda)$ represents a scaling factor which depends on the parameter $\lambda$ weighting the gradient penalty term. Finally, $\Omega \subset \mathbb{R}^{d}$ denotes the domain occupied by the whole system.

We investigate in detail the case of ad- and de-sorption of uniform particles [13] and then give a generalization towards mixtures of particles which even allow to account for surface active species, such as surfactants [14, 15]. We further extend the new free energy formulation towards incompressible and immiscible fluid flows. Our approach also provides a solid basis for extensions of studies on the formation and evolution of electrical double layers in electrochemistry [16, 17], with applications in energy storage devices such as supercapacitors, batteries, and micro-fluidic devices.

\section{Adsorption on dynamic interfaces}

In one dimension and for a fixed fluid-fluid interface at $x=0$ (in the sense of "Gibbs surface"), the following free energy

$$
\gamma^{e x}(c):=\int_{0}^{\infty} f_{b}^{e x}(c)+f_{I}(c) \delta_{0}(x) d x
$$

was studied by Andelman, Ariel, and Diamant [8,9] and by Mohrbach [18]. $\delta_{0}(x)$ stands for the Dirac delta function that is unity at $x=0$ and zero elsewhere. 
$c(x, t)$ represents a local volume fraction of particles in the immiscible two-phase fluid. and the variable $f_{b}^{e x}$ stands for the excess in bulk grand canoncial free energy density which is defined by the ideal entropy of mixing, i.e. $f_{b}^{e x}(c):=$ $\left(k_{B} T\left[c(\log c-1)-c_{b}\left(\log c_{b}-1\right)\right]-\mu_{b}\left(c-c_{b}\right)\right) / a^{3}$, where $a>0$ is the molecular dimension of the particles. $\mu_{b}$ and $c_{b}$ denote fixed equilibrium bulk chemical potential $\mu_{b}:=\lim _{x \rightarrow \infty} \mu(x)$ and fixed bulk volume fraction density of the particles $c_{b}:=$ $\lim _{x \rightarrow \infty} c(x)$, respectively. To properly account for the higher particle concentration at a fluid-fluid interface, the non-ideal entropy of mixing with an additional adsorption parameter $\alpha$, and interaction parameter $\beta$ is introduced by

$$
\begin{aligned}
f_{I}(c):= & \left(k_{B} T[c \log c+(1-c) \log (1-c)]\right. \\
& \left.-\alpha c-\beta / 2 c^{2}-\mu_{I} c\right) / a^{2}
\end{aligned}
$$

where the chemical potential $\mu_{I}$ is equal to the chemical potential of the solution next to the interface, i.e., $\mu_{I}:=\lim _{x \rightarrow 0} \mu(x)$. We note that $\beta$ accounts in an effective/averaged manner for local interactions between the particles and the immiscible fluids. This simplified formulation does not account for the geometry and evolution of the interface formed between the two immiscible fluids [19].

Here, we shall develop a generic theoretical and computational framework that accounts for both the particle densities and a dynamic immiscible interface which are nonlinearly coupled. Our framework builds also the basis for studying specific particles such as surfactants which can be accounted for by the interaction energy defined via $\beta$ in (2). First, we include the characterization of particles by the contact angle into the free energy (2), where the variable $c$ subsequently represents the density of particles for convenience. Based on the well-known free-energy change induced by primary mechanism of adsorption at the fluid-fluid interface $[1,6,20]$, we correspondingly write for a change in particle density

$$
\delta F_{a d s}:=-\gamma_{12} \pi(1-|\cos \theta|)^{2} \overline{\delta c}
$$

for adsorbing a single particle, where $\theta$ is the contact angle formed at the triple phase interface and $\gamma_{12}$ the contact angle formed between the particle-free immiscible fluid interface. Via a first-order Taylor approximation around equilibrium at the interface $I$, i.e., $\left.\left.\gamma^{e x}\left(c_{b}+\overline{\delta c}\right)\right|_{I} \approx \gamma^{e x}\left(c_{b}\right)\right|_{I}+\left.\frac{\delta \gamma^{e x}\left(c_{b}\right)}{\delta c} \overline{\delta c}\right|_{I}$, we can identify

$$
\delta F_{a d s}=\left.\left(\gamma^{e x}\left(c_{b}+\overline{\delta c}\right)-\gamma^{e x}\left(c_{b}\right)\right)\right|_{I}=\left.\frac{\delta \gamma^{e x}}{\delta c}\left(c_{b}\right)\right|_{I} \overline{\delta c}
$$

where $\overline{\delta c}$ is a small variation of the interfacial particle density. This finally allows us to define $\alpha$ for a given interaction energy $\beta$, which can be identified as the absolute value of the minimum energy of an associated inter-particle potential, e.g. of Lennard-Jones type. 


\subsection{Adsorption of uniform particles}

We now generalize (1) towards a diffuse interface formulation by introducing an additional order parameter $\phi$ based on the well-accepted Cahn-Hilliard approximation, i.e., $e(\phi):=W(\phi)+\lambda^{2}|\nabla \phi|^{2}$, where $W(s):=\left(1-s^{2}\right)^{2} / 4$ is the dimensionless double-well potential.

We note that $\lambda$ is proportional to the interfacial width and hence proportional to the molecular dimension $a$ and the particle concentration $c$. Variational considerations imply that $W(\phi)$ is only non-zero in a diffuse neighborhood around the interface. Therefore, we define the following free-energy density

$$
f(\phi, c):=e(\phi)+\mathrm{s}(\lambda) W(\phi) f_{I}(\phi, c)+\chi_{1}(\phi) f_{b}^{e x}(c),
$$

where $\mathrm{s}(\lambda):=3 \sqrt{2} / \lambda($ see also $[21,22])$ and

$$
f_{I}(\phi, c):=\left(k_{B} T\left[\log c+\frac{|\phi|+1}{2} \log \frac{|\phi|+1}{2}\right]-\alpha c-\beta c^{2}-\mu_{I} c\right) / a^{2},
$$

for $\frac{|\phi|+1}{2}=1-c$ and $\mu_{I}=\left.\mu\right|_{I}$ denotes again the chemical potential adjacent to the interface as in (2). The parameter $c_{b}$ stands for the bulk equilibrium density of particles, i.e., $c_{b}:=\frac{m_{\text {tot }}}{\mathcal{L}\left(\Omega_{1}\right)}$, where $m_{\text {tot }}$ is the total number of particles and $\mathcal{L}$ denotes the Lebesgue measure such that $\mathcal{L}\left(\Omega_{1}\right):=\int_{\Omega} \chi_{1}(\phi) d \mathbf{x}$ represents the area of phase $\phi=+1$. From [23], one can approximate the characteristic function for the interface by $\chi_{I}(\phi) \approx \frac{3 \sqrt{2}}{\lambda} W(\phi)$. The definition (5) is physically and mathematically motivated by the Modica-Mortola theorem [11] which relates the surface tension to the double-well potential $W$. Moreover, the energy density $e$ in (5) is known to represent the perimeter of the phase enclosed by the interface in the sharp interface limit $\lambda \rightarrow 0$. Furthermore, $\chi_{1}(\phi):=(\phi+1) / 2$ approximates the characteristic function of the phase $\phi=+1$ in which the bulk solid particles are diffusing in the sense of Brownian motion. This altogether allows us to introduce the new interfacial energy (i.e., interfacial tension which depends on the particle density) for arbitrary, dynamic, and higher dimensional interfaces by

$$
\bar{\gamma}^{e x}(\phi, c):=\int_{\Omega} f(\phi, c) d \mathbf{x}
$$

We note that the order parameter $\phi$ needs to be considered independently of the particle density $c$, despite the constraint $1-c=(|\phi|+1) / 2$, in view of the following underlying physical situation: the densities $c_{\alpha}$ and $c_{\beta}$ of species forming the immiscible fluid are much larger than the particle density $c$. That means, the phases $\alpha$ and $\beta$ are phase separated and one can reduce the number of variables by introducing $\phi:=\frac{c_{\alpha}}{c_{\alpha}+c_{\beta}}$. Finally, the change in the particle density $c$ is primarily governed by the status of the interface $\phi$ but the physical role of $\phi$ and $c$ is not the same. Due to this different physical role, we need to distinguish the processes associated with these two variables in our free energy modelling. The first variation of $(7)$ with respect to $c$ leads to the following 
(a)

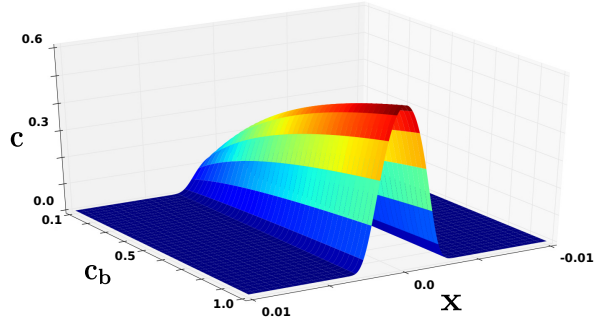

(c)

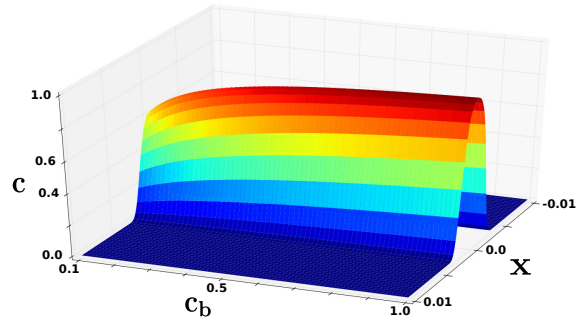

(b)

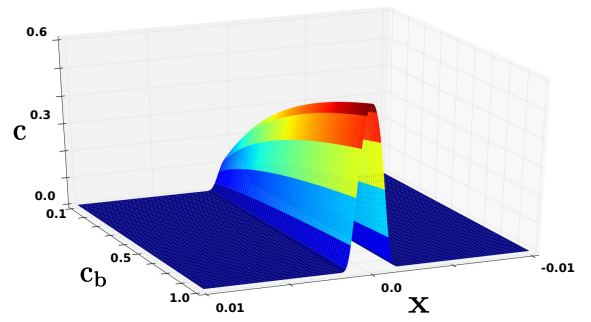

(d)

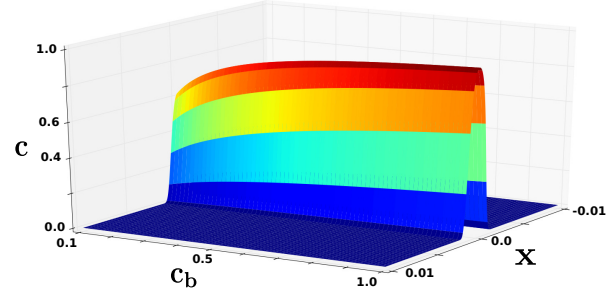

Figure 1. Generalized adsorption isotherm in (9) for $\beta=0, \phi_{\text {eq }}(x)=$ $\tanh (x /(2 \sqrt{2} \lambda))$, different interfacial widths $\lambda$, and different adsorption parameters $\alpha$. (a) $\alpha=0.5, \lambda=0.001$. (b) $\alpha=0.5, \lambda=0.0005$. (c) $\alpha=3.0, \lambda=0.001$. (d) $\alpha=3.0, \lambda=0.0005$.

chemical potential at the interface $I$,

$$
\begin{aligned}
\left.\mu_{c}^{e x}\right|_{I}:=\left.\frac{\delta \bar{\gamma}^{e x}}{\delta c}\right|_{I}= & \left(k_{B} T \log \frac{2 c}{|\phi|+1}-\alpha-\beta c\right. \\
& \left.-\mu_{I}\right)\left.\mathrm{s}(\lambda) W(\phi)\right|_{I} .
\end{aligned}
$$

In thermodynamic equilibrium we have that $\mu_{I}=\mu_{b}$ since the chemical potentials are everywhere equal to the bulk chemical potential $\mu_{b}=k_{B} T \log c_{b}$. Hence, the variation in the excess chemical potential vanishes, i.e., $\left.\mu_{c}^{e x}\right|_{I}=0$, and we obtain with (8) and $1-c=(|\phi|+1) / 2$ the new adsorption isotherm

$$
c=\left(\frac{c_{b}^{\mathrm{s}(\lambda) W(\phi)}}{c_{b}^{\mathrm{s}(\lambda) W(\phi)}+\exp \left[-(\alpha+\beta c) \mathrm{s}(\lambda) W(\phi) /\left(k_{B} T\right)\right]}\right)^{\frac{1}{\mathrm{~s}(\lambda) W(\phi)}},
$$

which also accounts for the state of the interior phase. If we neglect interactions, i.e., $\beta=$ 0 , and set $\mathrm{s}(\lambda)=1 / W(\phi)$ with $\phi=0$ (value on the interface), then (9) represents the classical Langmuir adsorption isotherm. Figure 1 depicts the adsorption isotherm (9) for an interface at $x=0$ and the well-known equilibrium profile $\phi_{e q}(x)=\tanh (x /(2 \sqrt{2} \lambda))$.

The expression in (7) can be interpreted as the free energy of the interface, which is located at $\mathbf{x} \in \Omega$ such that $\phi=0$, with particle concentrations $c$. We then derive evolution equations by the principle of steepest descent and its functional generalization 
to gradient flows. That means, we obtain the gradient flows

$$
\left\{\begin{aligned}
\frac{\partial \phi}{\partial t} & =\operatorname{div}\left(M_{\phi} \nabla\left(\frac{\delta \bar{\gamma}^{e x}}{\delta \phi}\right)\right), \\
\frac{\partial c}{\partial t} & =\operatorname{div}\left(c M_{c} \nabla\left(\frac{\delta \bar{\gamma}^{e x}}{\delta c}\right)\right),
\end{aligned}\right.
$$

where the equations are made dimensionless by the bulk diffusion time scales $\tau_{\phi}:=\frac{k_{B} T a^{3}}{M_{\phi}}$ and $\tau_{c}:=\frac{k_{B} T a^{3}}{M_{c}}$. We note that the mobilities $M_{\phi}$ and $M_{c}$ can be related to a scalar product with respect to which we measure the variational derivatives $\frac{\delta \gamma^{e x}}{\delta \phi}$ and $\frac{\delta \gamma^{e x}}{\delta \phi}$. It is straightforward to verify that $\phi$ and $c$ are conserved quantities after multiplying both sides in (10) by 1 , integrating over space $\Omega$, integration by parts on the right-hand side, and a final integration over time. It is also noteworthy that a new stochastic mode reduction strategy for gradient-flow systems was recently proposed in [25] and it allows for a rigorous dimensionally reduced description for such systems.

\subsection{Adsorption of different types of particles}

For simplicity, we state subsequent equations for identical molecular dimension $a$ for both types of particles $c_{1}$ and $c_{2}$ while noting that a generalization towards different molecular dimensions is straightforward. In order to account for mixtures of two (i.e., $c_{1}$ and $c_{2}$, whereas an according extension to an arbitrary but finite number of different types of particles is straightforward), we extend the interfacial free energy (2) as follows

$$
\begin{gathered}
f_{I}\left(c_{1}, c_{2}, \phi\right)=\frac{1}{a^{2}}\left(c_{1} \log c_{1}+c_{2} \log c_{2}+(|\phi|+1) / 2 \log (|\phi|+1) / 2-\epsilon_{12} c_{1} c_{2}\right. \\
\left.-\left(\alpha_{1}+\mu_{I}^{1}\right) c_{1}-\left(\alpha_{2}+\mu_{I}^{2}\right) c_{2}-\frac{\beta_{1}}{2} c_{1}^{2}-\frac{\beta_{2}}{2} c_{2}^{2}\right),
\end{gathered}
$$

where $(|\phi|+1) / 2:=1-c_{1}-c_{2}$ is the surface coverage of solvent represented by the order parameter $\phi$ (e.g. water/oil) and $\epsilon_{12}$ is the interaction energy between $c_{1}$ and $c_{2}$. We note that the total particle masses $m_{\text {tot }}^{(1)}$ and $m_{\text {tot }}^{(2)}$ are such that $m_{\text {tot }}^{(1)}+m_{\text {tot }}^{(2)}<1$.

$\mu_{I}^{1}$ and $\mu_{I}^{2}$ are chemical potentials of a subsurface layer in contact with the interface such that $\mu_{I}^{i} \neq \mu_{i}^{b}$ for $i=1,2$ out of equilibrium. But for simplicity, we will later on assume that $\mu_{I}^{i}=\mu_{i}^{b}$ for $i=1,2$ where $\mu_{i}^{b}$ represent the corresponding bulk chemical potentials. Based on (5) and (11), we then define the generalized surface tension $\bar{\gamma}^{e x}$ according to $(7)$, where $f_{b}^{e x}$ is canonically extended towards

$$
\begin{aligned}
f_{b}^{e x}\left(c_{1}, c_{2}\right)= & \left(k_{B} T\left[c_{1}\left(\log c_{1}-1\right)-c_{1}^{b}\left(\log c_{1}^{b}-1\right)+c_{2}\left(\log c_{2}-1\right)-c_{2}^{b}\left(\log c_{2}^{b}-1\right)\right]\right. \\
& \left.-\mu_{I}^{1}\left(c_{1}-c_{1}^{b}\right)-\mu_{I}^{2}\left(c_{2}-c_{2}^{b}\right)\right) / a^{3}
\end{aligned}
$$


Herewith, we formally obtain the two chemical potentials

$$
\begin{aligned}
\left.\mu_{1}^{e x}\right|_{I}:= & \frac{\delta \bar{\gamma}^{e x}}{\delta c_{1}}=\mathrm{s}(\lambda) W(\phi)\left[k_{B} T \log \frac{2 c_{1}}{|\phi|+1}-\beta_{1} c_{1}-\epsilon_{12} c_{2}-\left(\alpha_{1}+\mu_{I}^{1}\right)\right] \frac{1}{a^{2}} \\
& +\left.\chi_{1}(\phi)\right|_{I}\left[k_{b} T\left(\log c_{1}-1\right)-\mu_{I}^{1}\right] \frac{1}{a^{3}}, \\
\left.\mu_{2}^{e x}\right|_{I}:= & \frac{\delta \bar{\gamma}^{e x}}{\delta c_{2}}=\mathrm{s}(\lambda) W(\phi)\left[k_{B} T \log \frac{2 c_{2}}{|\phi|+1}-\beta_{2} c_{2}-\epsilon_{12} c_{1}-\left(\alpha_{2}+\mu_{I}^{2}\right)\right] \frac{1}{a^{2}} \\
& +\left.\chi_{1}(\phi)\right|_{I}\left[k_{b} T\left(\log c_{2}-1\right)-\mu_{I}^{2}\right] \frac{1}{a^{3}},
\end{aligned}
$$

where the characteristic functions for the bulk phase $\phi=+1$ vanish at the interface $I$, i.e., $\left.\chi_{1}(\phi)\right|_{I}=0$. At equilibrium, the excess chemical potentials (13) are set to zero by the assumption that the chemical potential is globally equal to the bulk values. This leads then to the adsorption isotherms,

$$
\begin{aligned}
& c_{1}=\left(\frac{\left(c_{1}^{b}\left(1-c_{2}\right)\right)^{\mathrm{s}(\lambda) W(\phi)}}{\left(c_{1}^{b}\right)^{\mathrm{s}(\lambda) W(\phi)}+\mathrm{e}^{-\left(\alpha_{1}+\beta_{1} c_{1}+\epsilon_{12} c_{2}\right) \mathrm{s}(\lambda) W(\phi) /\left(k_{B} T\right)}}\right)^{\frac{1}{\mathrm{~s}(\lambda) W(\phi)}}, \\
& c_{2}=\left(\frac{\left(c_{2}^{b}\left(1-c_{1}\right)\right)^{\mathrm{s}(\lambda) W(\phi)}}{\left(c_{2}^{b}\right)^{\mathrm{s}(\lambda) W(\phi)}+\mathrm{e}^{-\left(\alpha_{2}+\beta_{2} c_{2}+\epsilon_{12} c_{1}\right) \mathrm{s}(\lambda) W(\phi) /\left(k_{B} T\right)}}\right)^{\frac{1}{\mathrm{~s}(\lambda) W(\phi)}},
\end{aligned}
$$

where $c_{1}^{b}$ and $c_{2}^{b}$ are the equilibrium bulk densities. If we set $\mathrm{s}(\lambda)=1 / W(\phi)$ and the particle interactions $\beta_{i}=0, i=1,2$, as well as $\epsilon_{12}=0$, then we again end up with the Langmuir adsorption isotherm.

We also recover the same dynamic equations (10) after replacing $(10)_{2}$ by two corresponding equations, i.e., for the chemical potentials (13) instead of $\frac{\delta \bar{\gamma}^{e x}}{\delta c}$ introduced in (13).

\subsection{Generalization to incompressible fluids}

We adapt the variational principles in $[19,26]$ in order to generalize (a) and (b) to include fluid flow. In the context of porous media, related variational methods have been applied in [27]. We extend $\bar{\gamma}^{e x}$ to the action functional

$$
\begin{aligned}
& F\left(\mathbf{x}(\mathbf{X}, t)=\int_{0}^{T} \int_{\Omega}\left(\frac{1}{2}\left|\mathbf{x}_{t}(\mathbf{X}, t)\right|^{2}\right.\right. \\
& -\frac{\lambda^{2}}{2}\left|\hat{\mathrm{D}}^{-1} \nabla_{\mathbf{x}} \phi(\mathbf{x}(\mathbf{X}, t), t)\right|^{2}-\lambda^{2} W(\phi(\mathbf{x}(\mathbf{X}, t), t)) \\
& -\mathrm{s}(\lambda) W(\phi(\mathbf{x}(\mathbf{X}, t), t)) f_{I}(c(\mathbf{x}(\mathbf{X}, t), t)) \\
& \left.-\chi_{1}(\phi(\mathbf{x}(\mathbf{X}, t), t)) f_{b}^{e x}(c(\mathbf{x}(\mathbf{X}, t), t))\right) \operatorname{det} \hat{\mathrm{D}} d \mathbf{X} d t
\end{aligned}
$$

for the interfacial width $\lambda \propto$ (surface tension $) \times($ capillary width). We restrict ourselves to incompressible fluids and define the reference (Eulerian) coordinate $\mathbf{x}(\mathbf{X}, t)$ via the 

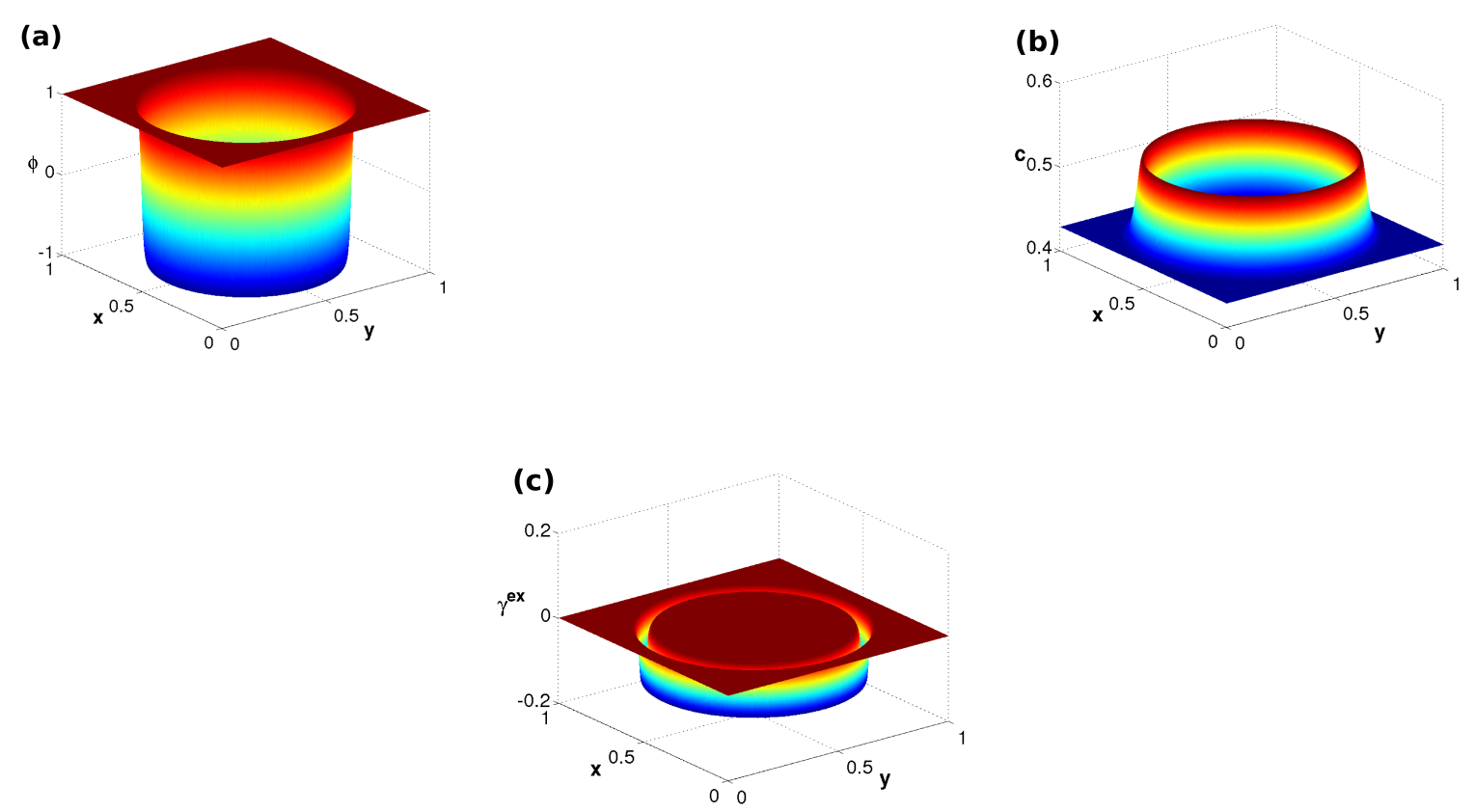

Figure 2. (a) Equilibrium phase field variable $\phi_{e q}(x)=\tanh \left(d_{r}(x) /(2 \sqrt{2} \lambda)\right)$ defined via the signed distance function $d_{r}(x)=\sqrt{\left(x-x_{0}\right)^{2}+\left(y-y_{0}\right)^{2}}-r$. (b) Generalized adsortion isotherm in Equation (9) with $c_{b}=0.75, \lambda=0.0005$, and $r=0.4$. (c) Free energy $\gamma^{e x}\left(c_{e q}, \phi_{e q}\right)$ for equilibrium particle concentration which is computed under an equilibrium phase field $\phi_{e q}$.

material (Lagrangian) coordinate $\mathbf{X}$, i.e.,

$$
\mathbf{x}_{t}(\mathbf{X}, t)=\mathbf{u}(\mathbf{x}(\mathbf{X}, t), t), \quad \mathbf{x}(\mathbf{X}, 0)=\mathbf{X},
$$

and $\hat{\mathrm{D}}(\mathbf{x}(\mathbf{X}, t), t)=\frac{\partial \mathbf{x}(\mathbf{X}, t)}{\partial \mathbf{X}}$ is th deformation tensor (strain) of the flow map [16]. The kinetic energy density $\frac{1}{2}\left|\mathbf{x}_{t}(\mathbf{X}, t)\right|^{2}$ leads to the Eulerian part of the incompressible Navier-Stokes equation. The terms arising due to the presence of particles, are $W^{\prime}(\phi) f_{I}(c) \nabla \phi, W(\phi) f_{I}^{\prime}(c) \nabla c$, and $\frac{1}{2} f_{b}^{e x}(c) \nabla \phi+\chi_{1}(\phi) f_{b}^{e x}(c) \nabla c$, such that we end up with the following momentum equation

$$
\left\{\begin{array}{l}
\frac{\partial \mathbf{u}}{\partial t}+\mathbf{u} \cdot \nabla \mathbf{u}-\Delta \mathbf{u}+\nabla p \\
=-\lambda^{2} \operatorname{div}(\nabla \phi \otimes \nabla \phi-W(\phi) \hat{\mathrm{I}}) \\
\quad-\mathrm{s}(\lambda) W^{\prime}(\phi) f_{I}(c) \nabla \phi-\mathrm{s}(\lambda) W(\phi) f_{I}^{\prime}(c) \nabla c \\
\quad-\frac{1}{2} f_{b}^{e x}(c) \nabla \phi-\chi_{1}(\phi) f_{b}^{e x^{\prime}}(c) \nabla c \\
\operatorname{div}(\mathbf{u})=0
\end{array}\right.
$$

where $\hat{\mathrm{I}}$ is the identity tensor. We note that the viscous term $-\Delta \mathbf{u}$ in equation [17] enters by the maximum dissipation principle (PMD) [28, 29, 30]. The variational framework (15) and (16) also extends the gradient flow (10) for the single and mixed particles to 

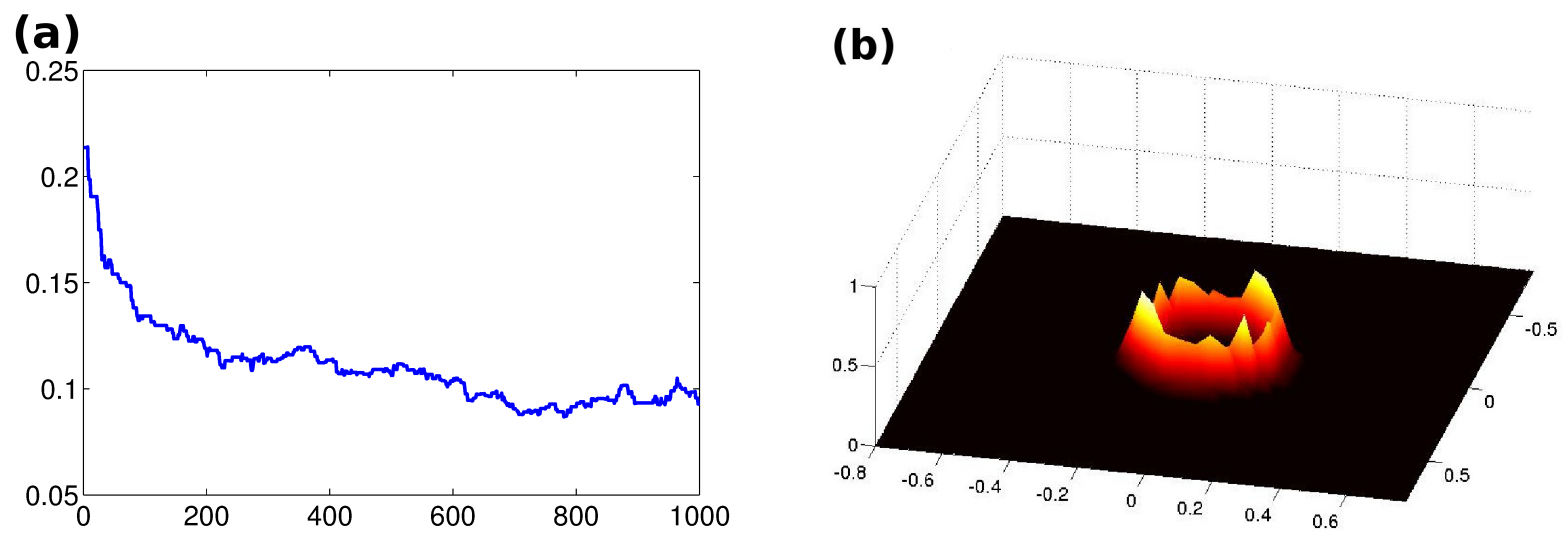

Figure 3. Computation with 275 particles. (a) Decay of interfacial tension as a result of minimizing the free energy (7). (b) Interfacial energy density.

fluid flow by the coupled partial differential equations (PDEs)

$$
\left\{\begin{array}{l}
\frac{\partial \phi}{\partial t}+\mathbf{u} \cdot \nabla \phi=\operatorname{div}\left(M_{\phi} \nabla\left(\frac{\delta F}{\delta \phi}\right)\right) \\
\frac{\partial c}{\partial t}+\mathbf{u} \cdot \nabla c=\operatorname{div}\left(c M_{c} \nabla\left(\frac{\delta F}{\delta c}\right)\right)
\end{array}\right.
$$

\section{Computations: Verification of decreasing interfacial tensions and surface pressure rise}

We scrutinize our modelling and computational framework with two model computations for equilibrium particle densities $c_{b}=\frac{m_{\text {tot }}}{\mathcal{L}\left(\Omega_{1}\right)}=\frac{275 m_{p}}{\mathcal{L}\left(\Omega_{1}\right)}$ and $c_{b}=\frac{350 m_{p}}{\mathcal{L}\left(\Omega_{1}\right)}$, where $m_{p}$ is the mass of a single particle. For simplicity, we neglect fluid flow. In Fig. 3 we depict computational results of characteristic quantities such as the interfacial tension, free energy, and particle configurations. We observe that the interfacial tension $\bar{\gamma}^{e x}(\phi, c)$ decreases with increasing particle density $c$, see (7), and also a non-uniform interfacial energy $e(\phi)$ due to the presence of particles on the interface and the dependence of phase field parameter $\phi$ on $c$. Herewith, we recover the primary experimental feature of a decreasing interfacial tension [Fig. 3 (a)] under the adsorption of particles as for instance demonstrated in [13]. Also in a second computation our computational framework captures the experimentally observed decrease of interfacial energy by an increase of the interfacial particle density [13], see Fig. 4 (a).

Finally, we could also recover the experimentally observed behaviour of the surface pressure $\Pi_{s}$ in dependence of the interfacial area $A_{s}$ as investigated in [24] for instance. The adsorption on a circular interface is shown in Fig. 5. Under these conditions and after setting (8) to zero, we obtain an expression for the equilibrium surface pressure $\left.\sigma\right|_{e q}-\left.\sigma_{A}\right|_{e q}=\Pi_{s}\left(\phi_{e q}, c_{e q}\right)=-\int_{\Omega} \mathrm{s}(\lambda) W\left(\phi_{e q}\right) f_{I}\left(\phi_{e q}, c_{e q}\right) d \mathbf{x}$, where $\sigma=\int_{\Omega} e(\phi) d \mathbf{x}$ is the microscopic surface tension and $\sigma_{A}:=\int_{\Omega} e(\phi)+\mathrm{s}(\lambda) W(\phi) f_{I}(\phi, c) d \mathbf{x}$ the macroscopic surface tension. Our formalism allows to qualitatively validate experimental results 
(a)

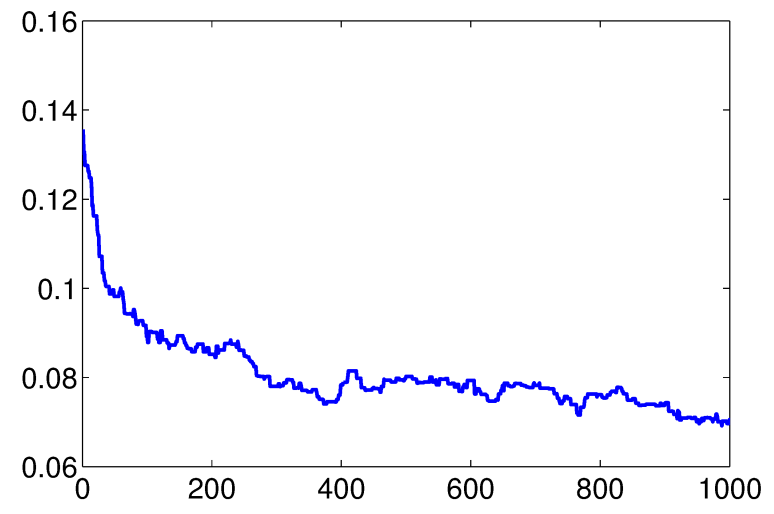

(b)

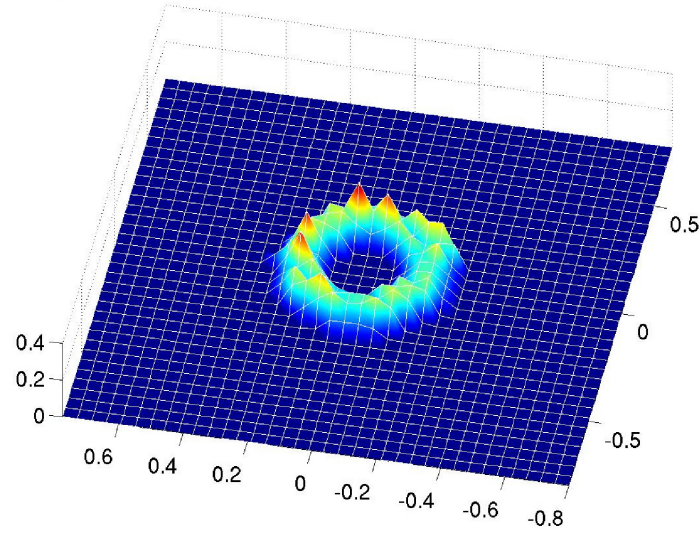

Figure 4. Computation with 350 particles. (a) Decay of the interfacial tension by minimizing (7). (b) Interfacial energy density.

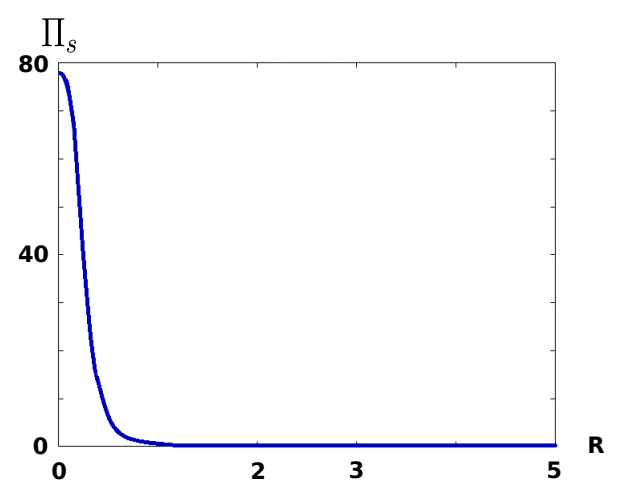

Figure 5. Surface pressure vs. surface area, i.e., $\Pi_{s}$ vs. $A_{s}$. We note that here the surface is $1 D$ as part of a two-dimensional domain $\Omega:=[-10,10]^{2} \subset \mathbb{R}^{2}$ and represents the circumference of a disk with phase $\phi=-1$, interface $\phi=0$, and the phase of the other immiscible fluid is $\phi=+1$. Hence, the $x$-coordinate is the radius $R$ as part of the circumference $A_{s}=2 \pi R$.

obtained in Aveyard et al. (2000) [24, Figure 2], see Fig. 5. We note that we employed the adsorption isotherm (9) for the computation of $\Pi_{s}$.

Herewith, we have a powerful tool which provides new research directions such as designing specific interfacial geometries by adding new interfaces at locations where the energy density is low. This demonstrates that we are able to compute the dynamic surface tension which depends on the interfacial particle density and geometry. Up to now, there has not been a formalism which considers the crucial influence of the interfacial geometry. We emphasize that a further novelty of our framework is that for dynamic interfaces we account for the wetting characteristics of the particles by the contact angle which determines the adsorption rate $\alpha$, see (2) and (4). As our methodology is based on a new free energy that incorporates all fundamental ingredients of particles' adsorption on dynamic interfaces, we can compute relevant 
physical quantities of experimental interest, such as the influence of the interfacial geometry and the particles' wetting properties on the adsorption rate, for instance.

\section{Conclusion}

We have outlined a new and general framework for particle adsorption on immiscible fluid interfaces by a thermodynamic free energy formulation leading to a new coupled system of PDEs. We further proposed a new efficient multi-level discretization strategy which accounts for the multi-scale feature of this complex multi-phase problem characterized by the presence of different length- and time-scales. Our formulation incorporates the characterization of the particles by the contact angle and captures the coupled-nonlinear dynamics between particle density and the interfacial location and geometry. The generality of our framework also enables to model surfactants, the incorporation of particles with non-local interactions [12, 31] or even of free energies obtained from first principles, such as density functional theory for instance, e.g. [32, 33, 34, 35], while of particular interest would be the extension of the framework to wetting problems, e.g. [36, 37, 38].

The strength of our methodology relies on its variational structure which allows to derive analytical expressions such as adsorption isotherms at thermodynamic equilibrium. These isotherms reduce in specific limits to the classical Langmuir isotherm for instance and generalize existing ones by taking the interfacial location and geometry into account. Our formulation also qualitatively validates relevant features experimentally observed in [13] and [24] for instance, that is, decreasing interfacial energies for increasing particle densities on the interface (Fig. 3 and Fig. 4) and the three distinct regions characterizing the behavior of the surface pressure in dependence of the interfacial area (Fig. 5), respectively.

In addition to these experimental agreements, we demonstrated the generality and flexibility of our framework by studying systems of mixed particles and even systems with fluid flow. This is a promising direction towards designing fluids with highly tunable transport properties. For instance, one could characterize conditions (e.g. fluid velocity, particle density, pressure, temperature, etc.) under which the particles are trapped on the interface and hence be transported between two locations. This should be of relevance in applications such as microfluidics and drug delivery.

These results offer a solid basis for the simulation and control of particle adsorption in a wide variety of physical phenomena and technological problems. The compatibility of our methodology with first-principle modelling strategies together with the scale resolving multi-level discretization approach, provides a promising tool to computationally exploiting complex particulate multiphase systems and their rich nature. 


\section{References}

[1] Aveyard R, Binks BP and Clint JH 2003 Adv. Colloid Interfac. 100-102 503-546 ISSN 00018686

[2] Koch K, Dew B, Corcoran TE, Przybycien TM, Tilton RD and Garoff S 2011 Mol. Pharm. 8 387-94 ISSN 1543-8392

[3] Yang XC, Samanta B, Agast SS, Jeong Y, Zhu ZJ, Rana S, Miranda OR and Rotello VM 2011 Angew. Chem. Int. Ed. Engl. 50 477-481

[4] Sun HQ, Zhang L, Li ZQ, Zhang L, Luo L and Zhao S 2011 Soft Matter 77601 ISSN 1744-683X

[5] Danov KD, Kralchevsky PA, Ananthapadmanabhan KP and Lips A 2006 Langmuir 22 106-15 ISSN 0743-7463

[6] Garbin V, Crocker JC and Stebe KJ 2012 J. Colloid Interf. Sci. 387 1-11 ISSN 1095-7103

[7] Maxwell IA, Kurja J, Van Doremaelea GHJ, German AL and Morrison BR 1992 Makromol. Chem. $1932049-2063$

[8] Ariel G, Diamant H and Andelman D 1999 Langmuir 15 3574-3581

[9] Diamant H and Andelman D 1996 Europhys. Lett. 34 575-580

[10] van der Sman R and van der Graaf S 2006 Rheologica Acta 46 3-11 ISSN 0035-4511 URL http://dx.doi.org/10.1007/s00397-005-0081-z

[11] Modica L and Mortola S 1977 Boll. Un. Mat. Ital. B 14 285-299

[12] Tcholakova S, Denkov ND and Lips A 2008 Phys. Chem. Chem. Phys. 10 1608-1627 ISSN 14639076

[13] Garbin V, Crocker JC and Stebe KJ 2012 Langmuir 28 1663-7 ISSN 1520-5827

[14] Nagarajan R 1991 Langmuir 7 2934-2969

[15] Diamant H and Andelman D 20043 Models of Gemini Surfactants Gemini Surfactants: Synthesis, Interfacial and Solution-Phase Behavior, and Applications Surfactant Science Series, vol. 117 ed Zana R and Xia J (Marcel Dekker, Inc.) pp 37-64

[16] Plieth W 2008 Electrochemistry for Materials Science (Elsevier) ISBN 978-0-444-52792-9

[17] Thomas-Alyea KE and Newman J 2010 Electrochemical Systems Third Edition (John Wiley \& Sons, Incorporated) ISBN 9780470880074

[18] Mohrbach H 2005 J. Chem. Phys. 123126101

[19] Liu C and Shen J 2003 Physica D: Nonlinear Phenomena 179 211-228 ISSN 01672789

[20] Pieranski P 1980 Phys. Rev. Lett. 45 569-573

[21] Wylock C, Pradas M, Haut B, Colinet C and Kalliadasis S 2012 Phys. Fluids 24032108

[22] Schmuck M, Pradas M, Pavliotis GA and Kalliadasis S 2012 Proc. R. Soc. A 468 3705-3724

[23] Elliott CM, Stinner B, Styles V and Welford R 2010 IMA J. Numer. Anal. 31 786-812 ISSN 0272-4979

[24] Aveyard R, Clint J, Nees D and Paunov V 2000 Langmuir 16 1969-1979

[25] Schmuck M, Pradas M, Kalliadasis S and Pavliotis GA 2013 Phys. Rev. Lett. 110244101

[26] de Gennes PG and Prost RL 1993 The Physics of Liquid Crystals (Oxford University Press)

[27] Schmuck M, Pradas M, Pavliotis G and Kalliadasis S 2013 Nonlinearity 263259 URL http: //stacks. iop.org/0951-7715/26/i=12/a=3259

[28] Svoboda J and Turek I 2006 Philos. Mag. B 64 749-759

[29] Hackl K and Fischer FD 2008 Proc. R. Soc. A 464 117-132 ISSN 1364-5021

[30] Hyon Y, Kwak DY and Liu C 2010 Discrete Cont. Dyn. S. 26 1291-1304 ISSN 1078-0947

[31] Zeng C, Brau F, Davidovitch B and Dinsmore AD 2012 Soft Matter 8 8582-8594 ISSN 1744-683X

[32] Evans DF and Wennerström H 1999 The Colloidal Domain: Where Physics, Chemistry, and Biology Meet (Wiley, New York)

[33] Yatsyshin P, Savva S and Kalliadasis S 2012 J. Chem. Phys. 136124113

[34] Goddard BD, Nold A, Savva N, Pavliotis GA and Kalliadasis S 2012 Phys. Rev. Lett. 109120603

[35] Yatsyshin P, Savva N and Kalliadasis S 2013 Phys. Rev. E 87 020402(R)

[36] Bonn D, Eggers J, Indekeu J, Meunier J and Rolley E 2009 Rev. Mod. Phys. 81 739-905

[37] Savva N, Kalliadasis S and Pavliotis GA 2010 Phys. Rev. Lett. 104084501 
General framework for adsorption processes on dynamic interfaces

[38] Savva N and Kalliadasis S 2011 Europhys. Lett. 9464004 